\title{
Diagrams in the Mind: Visual or Spatial?
}

\author{
Thomas Barkowsky \\ University of Bremen, SFB/TR 8 Spatial Cognition, \\ Enrique-Schmidt-Str. 5, 28359 Bremen, Germany \\ barkowsky@sfbtr8. uni-bremen.de
}

Diagrams are known to be powerful forms of knowledge representation both for spatial and non-spatial problems. For human reasoners their power results from their immediate accessibility through visual perception. This talk focuses on the mental side of diagrammatic representations. People coping with visual or spatial tasks use spatio-analogical mental representations. These representations are - like external diagrams - characterized by their structural correspondence with the state of affairs they represent. Usually, two forms of mental spatio-analogical representations are distinguished: spatial mental models are abstract representations that only focus on specific structural aspects of spatial relationships, whereas visual mental images are much more complete and more detailed representations.

However, what are the very differences between spatial mental models and visual mental images? Which conditions determine whether one form or the other is employed in a given reasoning tasks? What are the implications for the problem to be solved and how can the form of mental representation that is used be determined?

In general, for reasons of cognitive economy, representation structures that are easy to build up and easy to maintain are preferred over more complex ones. Thus, people prefer mental models over mental images, whenever the former are sufficient for dealing with a given task. In particular, humans are capable of successively integrating pieces of spatial knowledge into an existing mental representation making it more and more complex. This capability, which currently no technical AI system can satisfactorily simulate, may in a step-by-step manner render a spatial mental model into a mental image. Therefore, it is proposed to view mental models and mental images as the extremes of a continuous dimension rather than as two distinct classes of representations.

On the other hand, in reasoning about a given problem both spatial mental models and visual mental images can be induced in humans depending on how the task is communicated to them. Operating on visual mental images results in eye movements that resemble perceiving a real visual stimulus whereas operating on spatial mental models yields no correspondence of the structure of the mental representation and the eye gaze patterns. Thus, the eye movements of a human reasoner indicate whether mental models or mental images are involved in the reasoning process and also which aspects are in the focus when mental images are involved.

As a consequence, understanding and adopting the characteristics of mental representations on the one hand may help build more powerful and more flexible AI systems. On the other hand, a deeper understanding of mental spatial reasoning enables the design of intelligent interactive human-machine reasoning systems in which both partners efficiently work together in performing on a given task. 